\title{
Improved Computed Tomography Accuracy with a 1-mm versus 2- or 3-mm Slice Thickness for the Detection of Medial Coronoid Disease in Dogs
}

\author{
Raffaela T. Zweifel ${ }^{1}$ Pamela DiDonato ${ }^{1}$ Antje Hartmann ${ }^{2}$ Martin Kramer ${ }^{1}$ Kerstin H. von Pückler ${ }^{1}$ \\ ${ }^{1}$ Department of Veterinary Clinical Sciences, Clinic for Small Animals, \\ Justus Liebig University, Giessen, Germany \\ 2 Department of Diagnostic Imaging, Clinic for Small Animals \\ Hofheim, Hofheim, Germany \\ Address for correspondence Raffaela T. Zweifel, DVM, Dr. med. vet, \\ MRCVS, Department of Veterinary Clinical Sciences, Clinic for Small \\ Animals, Justus Liebig- University, Frankfurter Straße 108, 35392 Giessen, \\ Germany (e-mail: Raffaela.T.Zweifel@vetmed.uni-giessen.de).
}

Vet Comp Orthop Traumatol 2020;33:45-50.

\begin{abstract}
Keywords

- computed tomography

- canine elbow

- fragmented coronoid process

- fragmented medial coronoid

- elbow dysplasia

Objective Computed tomography (CT) is used complementarily to radiography for the evaluation of medial coronoid disease (MCD). We hypothesized that a slice thickness $>2 \mathrm{~mm}$ would significantly affect the image quality and detection of fragmentation of the medial coronoid process. This study aimed to assess CT features indicating direct and indirect evidence of MCD in 168 CT studies with slice thicknesses of 1-, 2- and $3 \mathrm{~mm}$.

Materials and Methods The CT studies were blinded in terms of CT slice thickness and patient data and randomly assessed by two independent observers. All dogs underwent arthroscopic evaluation of the elbow joints. Both observers were unaware of the arthroscopic findings.

Results Notably, blurring of the bone contour $(p=0.0001)$ was significantly influenced by slice thickness; here, a 1-mm thickness yielded a predominantly sharp and well-defined bone contour (observer 1, 91\%; observer 2, 79\%), whereas 2-(observer 1 , $39.3 \%$; observer $2,56.3 \%$ ) and especially $3-\mathrm{mm}$ slice thicknesses yielded blurred margins with significantly reduced sharpness (observer $1,0 \%$; observer $2,12.5 \%$ ). The 1-mm slice thickness also yielded the highest fragment detection rate (observer 1 , $55.4 \%$; observer $2,60.4 \%$ ). Furthermore, the detection of fragment positions and of single fragments and fissures differed substantially with slice thickness.

Clinical Relevance The findings of this study support the hypothesis that a CT slice thickness of $\geq 2 \mathrm{~mm}$ significantly affects fragment detection. In conclusion, a CT slice thickness of at least $1 \mathrm{~mm}$ is recommended for the assessment of MCD of the canine elbow.
\end{abstract}

\section{Introduction}

Fragmentation of the medial coronoid process (FCP) is a frequent sequela of medial coronoid disease (MCD) causing secondary osteoarthritic changes in the elbow joints of young dogs. ${ }^{1-4}$

Arthroscopy is widely considered the gold standard for MCD evaluation in lame dogs., ${ }^{5,6}$ Some authors hypothesize that fragmentation originates within the subchondral bone

and progresses secondarily into the cartilage. ${ }^{7}$ Subchondral defects may therefore be overlooked on direct arthroscopic examination unless cartilage is physically probed. However, this method is invasive.

The existing literature describes a combination of computed tomography (CT) and arthroscopy as the most adequate method for a complete evaluation of the medial coronoid process (MCP). ${ }^{5}$

(c) 2020 Georg Thieme Verlag KG Stuttgart · New York
DOI https://doi.org/ 10.1055/s-0039-3399524. ISSN 0932-0814.
May 7, 2019

accepted after revision

September 24, 2019

published online

November 22, 2019 
Computed tomography is a non-invasive diagnostic method with reported high levels of sensitivity (88.2\%) and specificity (84.6\%) for evaluating FCP. Therefore, CT is used complementarily to radiography in clinical cases with ambiguous radiographic findings and to detect early subchondral changes. ${ }^{8-10}$ The main benefit of $\mathrm{CT}$ is the capability to obtain a detailed and non-overlapping visualization of the subchondral bone. ${ }^{5,11,12}$ Accordingly, good CT image quality is required for an accurate assessment of the trabecular bone structure and subchondral bone contour of the MCP.

Computed tomography image quality depends on spatial resolution, artefacts and image noise. Here, slice thickness is inversely proportional to spatial resolution and image noise but proportional to the extent of blurring. With a greater slice thickness, small bone structures such as bone edges and bone contours are blurred and unsharp. With a smaller slice thickness, the bone structures are sharp but image noise may increase. The higher amount of image noise can be kept to a minimum by using a wide window setting and higher milliampère values. Previous reports have assumed that slice thickness affects $\mathrm{CT}$ image quality and the visibility of small fragments. ${ }^{5,6,13}$ Some authors have proposed the partial volume artefact as a main cause for reduced image quality. 6,14 Therefore, literature reports on evaluations of the elbow joint recommend a slice thickness of $<2 \mathrm{~mm}$ to increase spatial resolution and reduce slice thickness artifacts. ${ }^{15}$

In the authors' experience, the lack of an officially standardized $\mathrm{CT}$ protocol has led to the acquisition of $\mathrm{CT}$ studies with variable slice thicknesses across imaging centres. In some cases, a slice thickness of $\geq 2 \mathrm{~mm}$ results in misinterpretation of the data and missed MCP lesions. The International Elbow Working Group previously proposed breeding selection programs based on radiographic screening to exclude affected dogs. ${ }^{3,4}$ There has been an ongoing discussion about clinically applicable but reliable recommendations for CT settings during elbow scans in the context of breeding cases. To our knowledge, no published reports have investigated the influence of CT slice thickness on the evaluation of MCD in dogs.

The present study aimed to assess the effects of different slice thicknesses (1,2 and $3 \mathrm{~mm}$ ) on the detectability of different $\mathrm{CT}$ features of MCD of the canine elbow joint. We hypothesized that a slice thickness $>2 \mathrm{~mm}$ reduces image quality and further expected that a slice thickness of $\geq 2 \mathrm{~mm}$ yields different results in an evaluation of CT features, leading to false-negative diagnoses of MCD. The results of the present study potentially serve as a basis for recommendations regarding $\mathrm{CT}$ slice thickness in this context and may contribute to the validation of a prospective standardized CT scanning protocol for the evaluation of canine elbow joints.

\section{Materials and Methods}

\section{Selection and Description of Subjects}

This study is featured a retrospective design. Computed tomography datasets were obtained from a previous (unpublished) prospective study of the canine elbow joint that was conducted under approval of the Institutional Animal Care and Use Committee (GI 18/17-Nr. A19/2011). All own- ers consented to an inclusion of their data to this study. All dogs underwent orthopaedic and radiographic examinations, which depicted ambiguous features of MCD in at least one elbow joint. These dogs subsequently underwent simultaneous CT examination of both elbows. Datasets containing a complete depiction of the elbow joint were included. Afterwards, all dogs underwent arthroscopic examination of each limb performed by a board-certified specialist in orthopaedic surgery with more than 13 years of experience (C.P.). Dogs without arthroscopic examination of both forelimbs were excluded from the study. A board-certified veterinary radiologist with more than 11 years of experience (K.P.) and special expertise in musculoskeletal imaging made decisions regarding inclusion or exclusion.

\section{Computed Tomography Examination}

The dogs were examined under general anaesthesia with a combination of diazepam and levomethadon $(0.5 \mathrm{mg} / \mathrm{kg}$; KM diazepam-Ratiopharm 10 injection solution [Ratiopharm, Ulm, Germany] combined with 2-3 mg/kg KM L-Polamivet $2.5 / 0.125 \mathrm{mg} / \mathrm{mL}$ injection solution [Intervet, Deutschland $\mathrm{GmbH}$, Unterschleißheim, Germany]) and were positioned in dorsal recumbency for scanning. The thoracic limbs were arranged parallel and maximally extended cranially towards the gantry while fixed with bandages at the level of the carpal joint. The head was flexed caudolaterally. Both elbow joints were examined simultaneously.

All CT images were acquired using the same $\mathrm{CT}$ scanner (16-slice, Brilliance TM CT; Philips Medical Systems, Hamburg, Germany) and the same standardized CT protocol according to a previous study ${ }^{16}$ (helical mode, $140 \mathrm{kV}, 104$ $\mathrm{mAs}, 512 \times 512$ matrix, pitch 0.3 , high-resolution bone filter and bone algorithm). Transverse images were initially acquired at a 1-mm slice thickness. After scanning, reconstructed scans were produced at slice thicknesses of 2 and $3 \mathrm{~mm}$ (reconstruction interval -1 ). The scan margin included the olecranon up to $2 \mathrm{~cm}$ distal to the radial head.

\section{Assessment of Computed Tomography Features}

Computed tomography studies of 1-mm slice thickness, each reconstructed with 2- and 3-mm slice thickness, were reviewed on a computer screen (Apple iMac 2,3 GHz DualCore Intel Core i5, Apple Inc. Cupertino, California, United States) in a randomized order. All studies were evaluated independently by two observers with expertise in diagnostic imaging. The first observer was an intern in diagnostic imaging (R.Z.) and the second observer was the boardcertified veterinary radiologist (K.P.). Image data were assessed using a dedicated digital imaging and communications in medicine viewer (Osirix v.5.8.2; 32-bit; Pixmeo SARL Genf, Bern, Switzerland). The images were viewed in a multiplanar reconstruction and bone window (window width: +2500 Hounsfield units; window level: +500 Hounsfield units) after removing the patient data and imaged slice thickness from the display.

Evaluated structures within the elbow included the ulnar, radial and humeral bones. All CT images underwent initial subjective evaluation of the sharpness of the bone contours 
to determine whether image blurring permitted the accurate delineation. Accepted images were then assessed according to a structured protocol with special regard to $\mathrm{CT}$ features of MCD. The imaging features were subdivided as follows: (1) Imaging features indicating direct evidence of MCD: (a) presence or absence of FCP; (b) the number of visible fragments; (c) the type of fragments (i.e. non-displaced/displaced), where non-displaced fragments appeared as a thin hypoattenuating line that completely separated the tip of the MCP from its base; (d) the location of the detected fragments (i.e. apical, medial or radial to the MCP), when multiple fragments were identified, type and location were based on the majority of the fragments; (e) presence or absence of a kissing lesion (accompanied by subchondral sclerosis when severe); presence or absence of fissure lines of the MCP (an incomplete hypoattenuating line without separation of a fragment); (g) presence or absence of hinted fissure lines (a faint and short fissure line); (h) course of present fissure lines (i.e. cranioaxial, caudoabaxial, mid-MCP, apical to MCP); (2) Imaging features indicating indirect evidence of MCD: (a) shape of the MCP (i.e. round, peaked, irregular or flattened); (b) apical subchondral hypoattenuation of the MCP; (c) apical and medullary bone sclerosis of the MCP and sclerosis of the humeral trochlea, radial head or radial incisure; (d) regularity or irregularity of the contour of the bone radial incisures; (e) secondary arthritic changes evaluated at the dorsal aspect of the anconeal process; the MCP itself; at the ulnar and radial bone contours; at the radial head and incisure as well as the trochlea and capitulum of the humeral condyle.

\section{Statistical Analysis}

The statistical analysis was performed by a specialist in biomathematics and statistics (K.F.) of the Department of Biomathematics and Data Processing Justus-Liebig University Giessen. A commercially available statistics software program (1993; BMDP Statistical Software, Inc.; Los Angeles, California, United States) was used for the analysis. All scans with accepted image quality were evaluated and included in the statistical analysis. For frequency counting each imaging feature was encoded with a numerical value (e.g. simple features: presence $=1$; absence $=0$ : more complex features: apical $=1$, medial $=2$, or radial to the $M C P=3$.). The significance was set at a $p$-value $<0.05$.

To determine the effect of a slice thickness of 1,2 or $3 \mathrm{~mm}$ on the detectability of different CT features of MCD, the statistical analysis was subdivided. The binomial Wald Test (i.e. generalized-linear-mixed-model-estimation) was used to reveal any differences between the various slice thicknesses (1,2 and $3 \mathrm{~mm})$. A descriptive correlation analysis of the values representing the CT features assessed at each slice thickness was conducted, and the data were presented as a cross-tabulation. The McNemar Test was used to test for discordance between the values of each CT feature in pairs of slice thicknesses (i.e. 1 and $2 \mathrm{~mm}, 2$ and $3 \mathrm{~mm}$ and 1 and $3 \mathrm{~mm}$ ), and $95 \%$ confidence intervals were calculated to demonstrate the coincidence of the coupled values. Kappa statistics were used to test the agreement of the findings. Raw agreement and Cohen's kappa statistics were used to evaluate interobserver agreement and the effect of observer experience on CT image interpretation. The same methods were used for intermethod agreement of 1-mm thick CT slices (which normally provides the highest sharpness), with arthroscopic findings (i.e. the gold standard) regarding presence, type and number of fragments and fissures.

\section{Results}

\section{Study Population}

Twenty-eight client-owned dogs were presented with clinical signs of thoracic limb lameness at the Department of Veterinary Clinical Sciences at the Clinic for Small Animal Surgery Justus-Liebig University Giessen between 2012 and 2013. All dogs met the inclusion criteria. The sample comprised 18 male ( 7 neutered; 11 intact) and 10 female (4 neutered; 6 intact) dogs. Labrador Retrievers $(n=9)$ and Bernese Mountain Dogs $(n=5)$ were overrepresented in the sample population, which additionally included the following breeds: Irish Wolfhound $(n=1)$, Broholmer $(n=1)$, Elo $(n=1)$, Border Collie $(n=2)$, German Shepherd Dog $(n=1)$, Hollandse Herdershond $(n=1)$, Rottweiler $(n=1)$, Australian Cattle Dog $(n=1)$, Bordeaux $\operatorname{dog}(n=1)$, Entlebucher Mountain Dog $(n=1)$, Akita Inu $(n=1)$ and crossbreed dogs $(n=2)$. The dogs' body weight ranged from 15.5 to $58.5 \mathrm{~kg}$ (median, $35.1 \mathrm{~kg}$ ), and they ranged in age from 1 to 8 years (median, 4.5 years).

\section{Assessment of Computed Tomography Features}

For each dog one CT study (including both elbow joints) was acquired and two studies additionally reconstructed (three CT studies per elbow). A total of 168 CT studies were evaluated by observer 1 . Observer 2 excluded eight elbow joints due to lack of image quality.

1) Imaging features indicating direct evidence of MCD:

Fragment detection was significantly affected by slice thickness (observer 1: $p=0.0001$; observer 2: $p=0.00383$ ). Fragment detection rate was highest with a slice thickness of $1 \mathrm{~mm}(n=31 / 56$ [observer 1], $n=29 / 48$ [observer 2]). For both observers, the percentage of fragment detection decreased with increasing slice thickness ( $2 \mathrm{~mm}: n=24 / 56$ [observer 1] and $n=25 / 48$ [observer 2]; $3 \mathrm{~mm}: n=19 / 56$ [observer 1] and $n=20 / 48$ [observer 2]). For observer 2, fragments were reported with significant higher incidence for all slice thicknesses ( $p=0.00383$ ) ( - Fig. 1).

For observer 1 , the location of fragments significantly varied between different slice thicknesses: Out of 22 elbow joints with single or multiple fragments, apical fragments were most frequently identified with $1-\mathrm{mm}$ slice thickness ( $n=21 / 22, p=0.0003)$, followed by fragments located at the radial head $(n=15 / 22, p=0.0001)$ and medial to the MCP $(n=7 / 22, p=0.0001)$. At a $3-\mathrm{mm}$ slice thickness, fragments medial $(p=0.1025)$ and radial to the MCP $(p=0.0253)$ were more likely to be overlooked, compared with the $1-\mathrm{mm}$ slice thickness $(p=0.0001)$. Based on the number of detected fragments $(n=31)$, single fragments were predominantly 


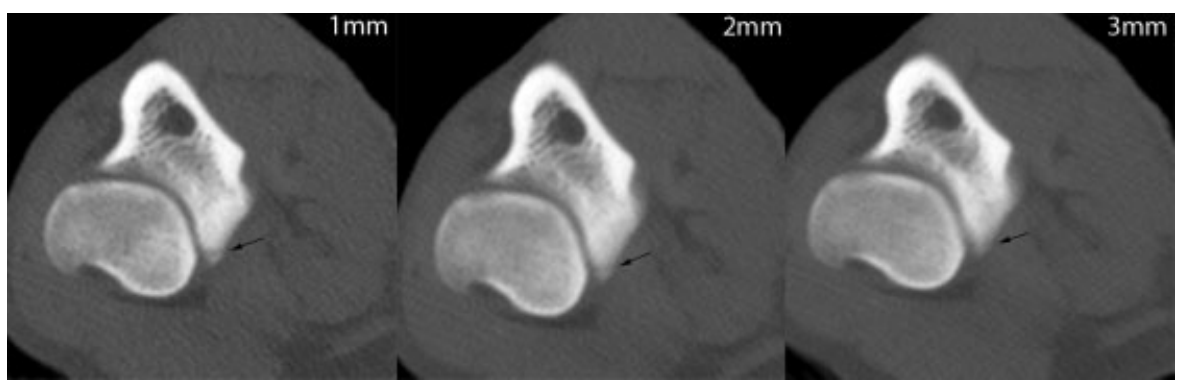

Fig. 1 Transverse computed tomography images obtained at 1-, 2- and 3-mm slice thicknesses (from left to right) at the level of the medial coronoid process of the canine elbow. The 1-mm slice shows a clearly delineated, thin, hypoattenuating fracture line (arrow) isolating the tip of the medial coronoid process. The fracture line becomes increasingly blurred and indistinct at a 2 -mm slice thickness. No fracture line is definable at a 3-mm slice thickness.

noted by observer 1 at a slice thickness of $1 \mathrm{~mm}(n=28 / 31$, $p=0.0001$ ). Single fragments were more likely to be overlooked with larger slice thickness ( $2 \mathrm{~mm}: n=21 / 31 ; 3 \mathrm{~mm}$ : $n=18 / 31$ ). However, no significant differences regarding the number of detected fragments were detected in the results by observer $2(p=0.056)$. Substantial differences were observed in the types of detected fragments (non-displaced/ displaced) for each slice thickness. In 28 elbows, non-displaced fragments were predominantly detected at a slice thickness of $1 \mathrm{~mm}(n=5 / 28), 2 \mathrm{~mm}(n=3 / 28)$ and $3 \mathrm{~mm}$ $(n=3 / 18)$. Therefore, non-displaced fragments were more likely to remain undetected at a slice thickness of 2 or $3 \mathrm{~mm}$. However, this finding was not significant $(p=0.102)$.

By observer 2, fissure lines were most frequently detected at a 1-mm slice thickness $(n=21 / 48)$ and with decreasing frequency at $2-\mathrm{mm}(n=14 / 48)$ and $3-\mathrm{mm}$ slice thicknesses $(n=15 / 48)$; although this finding was not significant $(p=0.0724)$. These fissures primarily coursed the tip of the $\operatorname{MCP}(n=4 / 7)$ and the radial head $(n=3 / 7)$. In particular, possible fissure lines were visible only in the 1 -mm-thick slices ( $n=3 / 28)$ (-Fig. 2).

2) Imaging features indicating indirect evidence of MCD: For each observer, significant differences for MCD contour were observed between different slice thicknesses $(p=0.0001)$. A sharp outline was more readily detected at a $1-\mathrm{mm}$ slice thickness (observer 1: $n=51 / 56$; observer 2: $n=38 / 48$ ), whereas sharpness was decreased at a 2-mm slice thickness (observer 1 : $n=22 / 56$; observer $2: n=27 / 48$ ) and particularly at a $3-\mathrm{mm}$ slice thickness (observer $1: n=56 / 56$; observer 2 : $n=42 / 48$ ).

The shape of the MCP did not differ significantly between different reconstructions. A highly significant difference was noted between a reduced bone density and the subjective evaluation of sclerosis of the MCP $(p=0.0001)$. Sclerosis of the MCP was most frequently noted at a $3-\mathrm{mm}$ slice thickness $(n=42 / 48)$ and at a focal location. Sclerosis of the humeral trochlea was less frequently noticed at slice thicknesses of $2 \mathrm{~mm}(n=27 / 31)$ and $3 \mathrm{~mm}(n=22 / 36)$ compared with $1 \mathrm{~mm}(n=29 / 31)$. The difference was significant when comparing 1-mm and 3-mm thick slices. Sclerosis of the radial head and radial incisure was rarely detected at any slice thickness. For observer $2, n=43 / 56$ of cases showed hypoattenuation of the MCP at a $1-\mathrm{mm}$ slice thickness and $n=37$ / 56 at a $2-\mathrm{mm}$ slice thickness. However, observer 1 reported hypoattenuated bone in $n=41 / 56$ of cases at a $3-\mathrm{mm}$ slice thickness $(p=0.0455)$, compared with $n=33 / 56$ at a $1-\mathrm{mm}$ slice thickness. Hypoattenuating bone lesions were almost exclusively evaluated with a focal distribution ( $1 \mathrm{~mm}: n=34$ / 34; $2 \mathrm{~mm}: n=34 / 34 ; 3 \mathrm{~mm}: n=33 / 37$ ).

Secondary osteoarthritic changes were recognized more frequently at a slice thickness of $3 \mathrm{~mm}$; however, this difference was not significant $(p=0.871)$. Interobserver disparity for MCP contour of the radial incisure was found. A smooth contour was determined most frequently at $3 \mathrm{~mm}$ (observer $1 ; n=25 / 53, p=0.00321)$ and $1 \mathrm{~mm}$ (observer $2 ;(n=13 / 15$, $p=0.00453$ ). An evaluation of kissing lesions revealed no significant differences between the slice thicknesses $(p=0.778)$. However, kissing lesions were less frequently observed at the $3-\mathrm{mm}$ slice thickness $(n=32 / 54)$, compared with 1 - and 2-mm slice thicknesses $(n=36 / 56$ for both observers).

Finally, the more experienced observer 2 found a consensus of $50 \%$ in an evaluation of $1-\mathrm{mm}$ slices from a CT

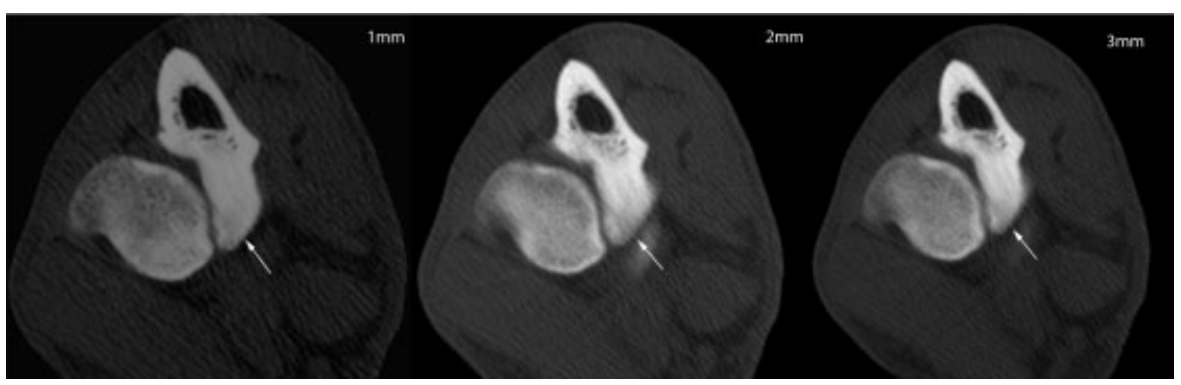

Fig. 2 Transverse computed tomography images obtained at 1-, 2- and 3-mm slice thicknesses (from left to right) at the level of the medial coronoid process of the canine elbow. The 1-mm slice shows a well-delineated, thin, hypoattenuating fissure line (arrow) running through the tip of the medial coronoid process. The fissure becomes indistinct at $2 \mathrm{~mm}$ and is invisible at a 3-mm slice thickness. 
examination with arthroscopic findings. Additionally, cases involving non-displaced fragments and/or fissure lines were more often detected with CT than with arthroscopy.

\section{Discussion}

To our knowledge, this is the first study to evaluate the influences of different $\mathrm{CT}$ slice thicknesses on the diagnostic reliability of CT features indicative of MCD in a clinical setting. Therefore, the present study contributes to and proves the necessity of at least $1-\mathrm{mm}$ CT protocols, for reliable diagnosis of MCD. Notably, the study findings support our initial hypothesis that a slice thickness $>2 \mathrm{~mm}$ would significantly reduce $\mathrm{CT}$ image quality, as a $1-\mathrm{mm}$ slice thickness produced the highest quality images, characterized by a well-defined and sharp bony contour. The difference between these images and those obtained at greater thicknesses was significant.

Moreover, the findings of this study support the hypothesis that a slice thickness of $\geq 2 \mathrm{~mm}$ would result in falsenegative diagnosis of MCD or fragmentation. Here, a 1-mm slice thickness yielded the highest detection rate of fragments, especially non-displaced and single fragments, and of fissures of the MCP; again, these differences were significant. Possible fissures were predominantly visible at the $1-\mathrm{mm}$ slice thickness, and the risk of missing FCP lesions was greatest at the 3-mm slice thickness. The likelihood of detecting fragments at the 2-mm and 3-mm slice thicknesses increased with the number of fragments. This finding is possibly explained by the larger area occupied by multiple fragments.

The present study confirmed that CT slice thicknesses of 2 and $3 \mathrm{~mm}$ correlated proportionally with blurring of small bone structures, edges and contours, particularly in the narrow joint spaces of the elbow. A previous study reported the highest spatial resolution with a slice thickness of $1 \mathrm{~mm} .{ }^{17}$ However, we note that the present study did not evaluate the effect of the slice thickness on conspicuity of MCP fragments of differing size. Other authors have reported that the presence of multiple fragments has been associated with a higher degree of osteophyte formation due to the advanced stage of the disease. ${ }^{16}$ Therefore, the reduced spatial resolution associated with an increased slice thickness could lead to the misinterpretation of osteophytes as MCP fragments and could contribute to an erroneous number of fragments in our study.

The interobserver discrepancies in the evaluation of secondary signs of MCD (reduced bone density, sclerosis, fissure lines and irregular radial incisures) may be attributed to subjectivity and differences in experience levels, as well as reduced image quality at slice thicknesses $>2 \mathrm{~mm}$. Differences in subjectivity among observers and the misinterpretation of stripe artefacts, appearing as hypoattenuating lines, have been discussed elsewhere., ${ }^{5,}$ Notably, a previously published study of Tromblee and colleagues has reported the effects of the CT display window and image plane on specific regions of the elbow joint; here, the transverse plane provided the greatest diagnostic cer- tainty for evaluations of the MCP and radial incisure, whereas the dorsal plane yielded the best diagnostic certainty for bone sclerosis and subchondral bone defects. ${ }^{18}$ The study also demonstrates the risk of misinterpretation of faint differences in attenuation in the MCP when evaluated subjectively. ${ }^{18}$ Objective bone density measurements using osteodensitometry or the use of visual grading scales may facilitate a more reliable evaluation of the trabecular bone density and reduce subjectivity.

In this study, no significant associations were observed between the slice thickness and the shape of the MCP or osteoarthritic changes.

The discrepancy between the findings of the $1 \mathrm{~mm}$ CT slices and arthroscopy was unexpected. In particular, the present study showed a greater detection rate of fissure lines and non-displaced fragments using CT. A recent study by Coppieters and colleagues compared CT and arthroscopic findings in canine elbow joints showing medial compartment syndrome and published comparable results. No significant agreement between the modalities concerning subchondral bone defects was found. Furthermore the presence of fragmentation of the MCP was not diagnosed consistently in all cases with arthroscopy but CT. ${ }^{19}$ This might be explained by the ability of detailed and non-overlapping visualization of the subchondral bone using $\mathrm{CT}^{5,11,12}$ However, several previous studies have stated that arthroscopy is mainly limited by the inability to visualize subchondral bone covered by intact overlying cartilage. ${ }^{4,17,20}$ Moreover, arthroscopic visualization, especially of small fragments, is limited by the narrow joint space of the elbow. ${ }^{4,21}$ The results of the present study provide support of the existing literature suggesting the combination of CT and arthroscopy as the most adequate method for a complete evaluation of the MCP. ${ }^{5}$ However, this issue is beyond the scope of the present study and requires further investigation.

The current study was limited primarily by the subjective assessment of CT features by a small number of observers with different experience levels. In practice, observer 2 reported no difference in the detection of single fragments between the different CT slice thicknesses. This suggests that observer experience may play an important role in the evaluation of primary subchondral changes, particularly when using larger slice thicknesses. The results of the present study could have been improved by achieving consensus opinions about the CT features among a larger number of experienced observers. Furthermore, the statistical findings could have benefited from including a larger number of dogs. It also remains unclear whether the faint subchondral bone defects and hypoattenuating lines visible at a 1-mm CT slice thickness were overinterpreted as FCP lesions or not visible arthroscopically because of the intact overlying cartilage layer. The discrepancy between the CT and arthroscopic findings in the present study reflects a main limitation in literature reports, namely the lack of a gold standard for the evaluation of the canine elbow joint pertaining a diagnosis of MCD especially in dogs younger than 12 months. Previously, another research group reported that micro-CT would be an ideal reference modality for evaluating 
the subchondral bone, especially in growing young dogs without clinical signs of lameness. ${ }^{5}$ We note, however, that micro-CT is considered an in vitro method. This shows a third limiting factor of our study: the population included only clinically lame adult dogs (older than 12 months) and no control group was introduced. Additional studies comparing CT slice thicknesses $<1 \mathrm{~mm}$ with histologic findings in clinically lame and in healthy dogs would be beneficial to clarify the discrepancies between CT and arthroscopy. Furthermore, it would be interesting to evaluate whether the use of CT with a slice thickness of less than $1 \mathrm{~mm}$ is required to diagnose the early non-clinical stages of MCD and to determine the impact of image noise on the detectability of MCD.

The results of the present study are generalizable to other clinical settings because all clinical scanners can be used to achieve a 1-mm slice thickness. Furthermore, comparable results with other dog breeds of comparable size and weight are likely. Therefore, the dog breed and weight were not considered significant factors. However, we might assume that a higher resolution is needed for an exact assessment of the elbow in a smaller dog.

In summary, the current study demonstrates that a slice thickness of $1 \mathrm{~mm}$ is beneficial for the detection of fissure lines, possible fissure lines and non-displaced fragments in comparison to 2 - and $3 \mathrm{~mm}$. Therefore, we recommend the use of a slice thickness of $\leq 1 \mathrm{~mm}$ for the CT scans intended to assess MCD in the canine elbow. Additionally, these findings provide suggestions for a standardized CT protocol.

\section{Note}

Portions of this study were published as an abstract and presented at the 2015 Annual Scientific Meeting of the International Veterinary Radiology Association Meeting, Fremantle, Western Australia.

\section{Authors' Contributions}

All authors contributed to conception of study, study design, acquisition of data and data analysis and interpretation. All authors also drafted, revised and approved the submitted manuscript.

\section{Funding}

None.

\section{Conflict of Interest \\ None declared.}

\section{Acknowledgments}

Authors would like to thank Dr. Klaus Failing (K.F.), Marion Sparenberg, Dr. Christine Peppler (C.P.) for collaboration in the current study.

\section{References}

1 Grøndalen J, Grøndalen T. Arthrosis in the elbow joint of young rapidly growing dogs. V. A pathoanatomical investigation. Nord Vet Med 1981;33(01):1-16
2 Temwichitr J, Leegwater PA, Hazewinkel HA. Fragmented coronoid process in the dog: a heritable disease. Vet J 2010;185(02):123-129

3 Janutta V, Hamann H, Klein S, Tellhelm B, Distl O. Genetic analysis of three different classification protocols for the evaluation of elbow dysplasia in German shepherd dogs. JSmall Anim Pract 2006;47(02):75-82

4 Flückiger M. Ellbogendysplasie (ED) beim Hund [Elbow dysplasia in the dog]. Schweiz Arch Tierheilkd 1992;134(06):261-271 In German

5 Villamonte-Chevalier A, van Bree H, Broeckx B, et al. Assessment of medial coronoid disease in 180 canine lame elbow joints: a sensitivity and specificity comparison of radiographic, computed tomographic and arthroscopic findings. BMC Vet Res 2015;11:243

6 Moores AP, Benigni L, Lamb CR. Computed tomography versus arthroscopy for detection of canine elbow dysplasia lesions. Vet Surg 2008;37(04):390-398

7 Danielson KC, Fitzpatrick N, Muir P, Manley PA. Histomorphometry of fragmented medial coronoid process in dogs: a comparison of affected and normal coronoid processes. Vet Surg 2006;35 (06):501-509

8 Carpenter LG, Schwarz PD, Lowry JE, Park RD, Steyn PF. Comparison of radiologic imaging techniques for diagnosis of fragmented medial coronoid process of the cubital joint in dogs. J Am Vet Med Assoc 1993;203(01):78-83

9 Reichle JK, Snaps F. The elbow. Clin Tech Small Anim Pract 1999;14 (03):177-186

10 Lau SF, Theyse LF, Voorhout G, Hazewinkel HA. Radiographic, computed tomographic, and arthroscopic findings in Labrador Retrievers with medial coronoid disease. Vet Surg 2015;44(04):511-520

11 Rau FC, Wigger A, Tellhelm B, et al. Observer variability and sensitivity of radiographic diagnosis of canine medial coronoid disease. Tierarztl Prax Ausg K Kleintiere Heimtiere 2011;39:313-322.Cook CR, Cook JL. Diagnostic imaging in canine elbow dysplasia: a review. Vet Surg 2009;38:144-153

12 Lau SF, Wolschrijn CF, Hazewinkel HA, Siebelt M, Voorhout G. The early development of medial coronoid disease in growing Labrador retrievers: radiographic, computed tomographic, necropsy and micro-computed tomographic findings. Vet J 2013;197(03):724-730

13 Kunst CM, Pease AP, Nelson NC, Habing G, Ballegeer EA. Computed tomographic identification of dysplasia and progression of osteoarthritis in dog elbows previously assigned OFA grades 0 and 1 . Vet Radiol Ultrasound 2014;55(05):511-520

14 Goldman LW. Principles of CT: radiation dose and image quality. J Nucl Med Technol 2007;35(04):213-225, quiz 226-228

15 Cook CR, Cook JL. Diagnostic imaging of canine elbow dysplasia: a review. Vet Surg 2009;38(02):144-153

16 Walde I, Tellhelm B. Fragmented medial coronoid process of the ulna (FCP) and osteochondrosis dissecans (OCD) of the canine elbow-joint and hock-joint-literature-review, diagnosis and therapy. Wien Tierarztl Monatsschr 1991;78:414-424

17 Groth AM, Benigni L, Moores AP, Lamb CR. Spectrum of computed tomographic findings in 58 canine elbows with fragmentation of the medial coronoid process. J Small Anim Pract 2009;50(01):15-22

18 Tromblee TC, Jones JC, Bahr AM, Shires PK, Aref S. Effect of computed tomography display window and image plane on diagnostic certainty for characteristics of dysplastic elbow joints in dogs. Am J Vet Res 2007;68(08):858-871

19 Coppieters E, Van Ryssen B, van Bree H, et al. Computed tomographic findings in canine elbows arthroscopically diagnosed with erosion of the medial compartment: an analytical method comparison study. Vet Radiol Ultrasound 2016;57(06):572-581

20 Van Ryssen B, van Bree H. Arthroscopic findings in 100 dogs with elbow lameness. Vet Rec 1997;140(14):360-362

21 Vermote KA, Bergenhuyzen AL, Gielen I, van Bree H, Duchateau L, Van Ryssen B. Elbow lameness in dogs of six years and older: arthroscopic and imaging findings of medial coronoid disease in 51 dogs. Vet Comp Orthop Traumatol 2010;23(01):43-50 